\title{
Activités
}

15-1 | 2018

Mettre en scène l'activité : les nouvelles figures de la simulation

\section{La qualité, l'innovation et la créativité du travail au sein des organisations : vers une politique globale de prévention de la souffrance psychique au travail}

Thèse de doctorat en psychologie. Mention : Psychologie du travail et des organisations. Soutenue publiquement le 27 octobre 2017 Ecole Doctorale : Sciences de l'éducation, psychologie, information et communication.

Institut de Psychologie. Laboratoire : Groupe de Recherche en

Psychologie Sociale

\section{Nadège Guidou}

\section{(2) OpenEdition}

\section{Édition électronique}

URL : http://journals.openedition.org/activites/3158

DOI : 10.4000/activites.3158

ISSN : 1765-2723

Éditeur

ARPACT - Association Recherches et Pratiques sur les ACTivités

\section{Référence électronique}

Nadège Guidou, «La qualité, l'innovation et la créativité du travail au sein des organisations : vers une politique globale de prévention de la souffrance psychique au travail », Activités [En ligne], 15-1 | 2018, mis en ligne le 15 avril 2018, consulté le 19 avril 2019. URL : http://journals.openedition.org/ activites/3158 ; DOI : 10.4000/activites.3158

\section{Ce document a été généré automatiquement le 19 avril 2019}

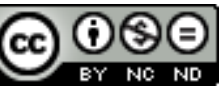

Activités est mis à disposition selon les termes de la licence Creative Commons Attribution - Pas d'Utilisation Commerciale - Pas de Modification 4.0 International. 


\section{La qualité, l'innovation et la créativité du travail au sein} des organisations : vers une politique globale de prévention de la souffrance psychique au travail

Thèse de doctorat en psychologie. Mention : Psychologie du travail et des organisations. Soutenue publiquement le 27 octobre 2017 Ecole Doctorale : Sciences de l'éducation, psychologie, information et communication.

Institut de Psychologie. Laboratoire : Groupe de Recherche en Psychologie Sociale

Nadège Guidou

\section{RÉFÉRENCE}

Nadège GUIDOU (2017), La qualité, l'innovation et la créativité du travail au sein des organisations : vers une politique globale de prévention de la souffrance psychique au travail. Thèse pour le doctorat en psychologie. Lyon : Lyon 2

\section{NOTE DE L'AUTEUR}

Jury de thèse :

Isabelle HANSEZ, Professeure, Université de Liège, Belgique, Rapporteuse Gérard VALLERY, Professeur des universités, Université de Picardie, Rapporteur Adalgisa BATTISTELLI, Professeure des universités, Université de Bordeaux, Examinatrice 
Dominique LHUILIER, Professeure émérite, CNAM Paris, Examinatrice

Philippe SARNIN, Professeur des universités, Université Lyon 2, Directeur de thèse.

1 L'intervention en prévention de la santé psychique au travail vise à transformer durablement les situations de travail. Elle nécessite donc d'agir en faveur d'une organisation du travail capacitante soutenant la création ou le renforcement d'espaces permettant de débattre du travail et d'agir sur l'environnement (Falzon, 2009). Pour autant, bien que de nombreux travaux aient abordé, par des perspectives diverses, ce passage à la transformation, on peut encore mettre perspective les divers modèles au regard de leurs ressemblances et différences mais aussi au regard des différents enjeux du terrain, qu'il s'agisse du contexte d'intervention ou encore des acteurs de la situation. Notre recherche vise donc à modéliser le passage de la connaissance (du diagnostic) à la transformation (l'action) en adoptant le point de vue des acteurs de la situation de travail. Dirigeants, managers et salariés jouent en effet un rôle déterminant dans ce passage qu'il s'agisse de l'analyse de l'activité, de l'élaboration des axes de transformation ou encore de l'appropriation des changements.

2 Dans un premier temps, nous identifions puis étudions différents modèles d'intervention remarquables pour leur articulation entre un cadre théorique solide autour de la dynamique santé-travail (connaissance) et un cadre méthodologique d'intervention (action): le modèle d'enquête qualitative (Dejours, 2015; Molinier, 2012; Dejours, \& Bègue, 2009), le modèle d'enquête quantitative simple (Ponnelle, \& coll., 2012 ; INRS, 2013), le modèle d'enquête participative mixte (Jeoffrion, \& coll., 2014 ; Petit, \& coll., 2011), le modèle de médiation par l'activité (Lhuilier, 2013 ; Amado, \& Lhuilier, 2012), le modèle ergonomique (Petit, \& coll., 2011 ; Caroly, 2010 ; Weill-Fassina, \& Rabartel, 2010 ; Hubault, 2009), le modèle développemental du métier (Miossec, \& Clot, 2011 ; Clot, 2011 ; Clot, \& Leplat, 2005), le modèle d'ingénierie de la discussion (Detchessahar, \& coll., 2015 ; Detchessahar, 2013 ; Grevin, 2011), le modèle systémique (Althaus, \& coll., 2013 ; Favaro, 2004 ; Benoit, \& Perez-Benoit, 2006 ; Checkland, 2000) et enfin le modèle institutionnel (Mendel, 1992; Rueff-Escoubès, 2008). Une seconde étape consiste en l'analyse des interactions observables entre ces divers processus d'intervention et les acteurs internes : nous avons en effet cherché à comprendre quelle activité les acteurs internes sont amenés à réaliser sur leur activité et leur organisation du fait - et au travers - de l'intervention. En procédant par une revue narrative et en nous situant dans le cadre épistémologique de la théorie ancrée, nous proposons ainsi trois dimensions susceptibles de théoriser le passage du diagnostic à la transformation et de soutenir l'activité à déployer par les acteurs internes dans ce passage : l'objet d'intervention, c'est-à-dire l'objet autour duquel l'activité des acteurs internes se porte durant l'intervention; le niveau d'implication des acteurs, c'est-à-dire les divers espaces de participation et les types de coopération à l'œuvre et enfin le processus cognitif de prévention implanté, c'est-à-dire le type de raisonnement engendré chez les acteurs dans l'élaboration des axes de transformation. Après une première enquête exploratoire par questionnaire visant à identifier les caractéristiques générales des interventions conduites sur le terrain des Services de Santé au Travail (SST) au regard de ces interrogations, deux recherche-action sont présentées. Chacune étudie les effets des diverses articulations testées entre les trois dimensions, en observant finement les activités déployées par les acteurs internes au fil du processus d'intervention.

3 L'ensemble de notre démarche s'inscrivant dans le cadre de la recherche fondamentale de terrain telle que décrite par Clot (2008), nous aboutissons dans notre dernière étude, à la 
proposition et l'expérimentation d'un nouveau modèle d'intervention permettant de dépasser les limites observées dans la littérature. Inscrite au sein d'un processus d'innovation organisationnelle, cette démarche soutient l'implication active des dirigeants supérieurs durant l'ensemble du processus, permettant ainsi de soutenir la dialectique entre organisation formelle et organisation vivante (Terssac, \& Lompré, 1996). La controverse professionnelle y est déployée au sein d'espaces dialogiques mêlant dirigeants supérieurs, managers et salariés et se construit à partir d'une analyse du travail réel utilisée comme support au déclenchement d'activités transitionnelles au sens de Winnicott (1971). Se faisant, les acteurs internes sont soutenus dans leurs travaux en développant des activités transitionnelles sur leur propre activité et organisation.

Finalement, ce travail nous amène, en plus d'ouvrir vers de nouvelles orientations scientifiques et pratiques, à questionner la posture de l'intervenant agissant en prévention de la santé psychique au travail : en déplaçant notre regard du côté des interactions entre les acteurs internes et le processus d'intervention, nous avons considéré le passage à la transformation au travers de l'activité ainsi exigée de la part des acteurs internes. Se faisant, nous considérons nécessaire de se positionner du côté de l'accompagnement de ces acteurs et dans le soutien et le développement de cette activité dans et par l'intervention.

\section{BIBLIOGRAPHIE}

Althaus, V. (2013). Élaboration et mise en œuvre d'une démarche d'intervention systémique pour les PME : construction théorique et application pratique dans cinq entreprises (Thèse de doctorat, Université de Lorraine à Nancy, France). Récupéré de docnum, l'archive de publication électronique de l'Université de Lorraine : http://docnum.univ-lorraine.fr/public/ DDOC_T_2013_0299_ALTHAUS.pdf.

Amado, G., \& Lhuilier, D. (2012). L'activité au cœur de l'intervention psychosociologique. Bulletin de psychologie, 519, $263-276$.

Benoit, D., \& Perez-Benoit, F. (2006). L'intervention systémique brève. Pour une application, hors champ thérapeutique, du modèle de résolution de problèmes développé en Thérapie. Revue Internationale de Psychosociologie, 26, 97-141.

Bertrand, K., L'Espérance, N., \& Flores Aranda, J. (dir). La méthode de la revue systématique : Illustration provenant du domaine de la toxicomanie des troubles mentaux concomitants chez les jeunes. Méthodes qualitatives, quantitatives et mixtes dans la recherche en sciences humaines, sociales et de la santé (p. 145-163). Quebec : PUQ.

Caroly, S. (2010). L'activité collective et la réélaboration des règles : des enjeux pour la santé au travail ( habilitation à diriger des recherches, Université Bordeaux 2 à Bordeaux, France). Récupéré de HAL, l'archive de publications électroniques : http://tel.archives-ouvertes.fr/docs/00/46/50/89/ PDF/HDR_Caroly.pdf

Checkland, P. B. (2000). Soft Systems Methodology: A Thirty-Year Retrospective. System Research and Behavioral Science, 17, 11-58. 
Clot, Y. (2011-b). Théorie en clinique de l'activité. Dans Maggi, B. Interpréter l'agir. Un défi théorique (p. 17-39). Paris : PUF.

Clot, Y. (2008). La recherche fondamentale de terrain : une troisième voie. Education permanente, 177, 67-78.

Clot, Y., \& Leplat, J. (2005). La méthode clinique en ergonomie et en psychologie du travail. Le Travail Humain, 4, 289-313.

Dejours, C. (2015-a). La méthodologie en psychopathologie du travail. Travailler, 35, 127-144.

Dejours, C., \& Bègues, F. (2009). Suicide et travail : que faire ? Paris : PUF.

Detchessahar, M. (2013). Faire face aux risques psycho-sociaux : quelques éléments d'un management par la discussion. Négociations, 19, 57-80.

Detchessahar, M., Gentil, S., Grevin, A., \& Stimec, A. (2015). Quels modes d'intervention pour soutenir la discussion sur le travail dans les organisations? Réflexions méthodologiques à partir de l'intervention dans une clinique. @GRH, 16, 63-89.

Favaro, M. (2004). Interventions thérapeutiques et consultance en entreprise. Hygiène et Sécurité du Travail, 195(2), 95-99.

Grevin, A. (2011). Les transformations du management des établissements de santé et leurs impacts sur la santé au travail. L'enjeu de la reconnaissance de la dynamique du don. Etude d'un centre de soins de suite et d'une clinique privée malade de "gestionnite" (thèse de doctorat, Université de Nantes à Nantes). Récupéré à l'archive de publications électroniques de l'Université de Nantes : http:// archive.bu.univ-nantes.fr/pollux/show.action?id=ff3a12f6-40ab-449c-a45d-17643676f117 Hubault, F. (2009). L'approche ergonomique des questions santé/travail. Mouvement, 58, 97-102.

INRS. (2013). Le point des connaissances sur le stress au travail. Récupéré le 14/06/2016 : http:// www.inrs.fr/media.html?refINRS=ED\%205021

Jeoffrion, C., Hamard, J.P., Barre, S., \& Boudoukha, A. H. (2014). Diagnostic organisationnel et prévention des risques psychosociaux dans un établissement d'accueil pour personnes âgées : l'intérêt d'une méthodologie mixte et participative. Le Travail Humain, 4, 373-399.

Lhuilier, D. (2013). Introduction à la psychosociologie du travail. Nouvelle Revue de Psychosociologie, $15,11-30$.

Miossec, Y., \& Clot, Y (2011). Le métier comme instrument de protection contre les risques psychosociaux au travail. Le cas des ingénieurs de proximité. Le Travail Humain, 74 (4), 341-363.

Molinier, P. (2012). Une enquête de psychodynamique du travail dans un département de recherche industrielle. Méthodologie, élaboration, résultat. Bulletin de psychologie, 3, 201-225.

Petit, J., Dugué, B., \& Daniellou, F. (2011). L'intervention ergonomique sur les risques psychosociaux dans les organisations : enjeux théoriques et méthodologiques. Le Travail Humain, 4, 391-409.

Ponnelle, S., Vaxevanoglou, X., \& Garcia, F. (2012). L'usage des outils d'évaluation du stress au travail : perspectives théoriques et méthodologiques. Le Travail Humain, 75, 179-213.

Terssac, G. de., \& Lompré, N. (1996). Pratiques organisationnelles dans les ensembles productifs : essai d'interprétation. Dans Spérandio, J.C. (dir.), L'Ergonomie face aux changements technologiques et organisationnels du travail humain (p.51-70). Toulouse : Octarès.

Weill-Fassina, A., \& Rabardel, P. (2010). Point de vue ergonomique sur les facteurs psychosociaux de risques pour la santé. Revue de la littérature réalisée dans le cadre des travaux du Collège 
d'expertise sur le suivi statistique des risques psychosociaux au travail. Récupéré le 03/04/2014 : http://www.college-risquespsychosociaux-travail.fr/site/Revue-Ergonomie.pdf

Winnicott, D W. (1971). Jeu et réalité. Paris : Gallimard.

\section{AUTEURS}

NADÈGE GUIDOU

Université Lumière Lyon 2, Laboratoire GRePS, 5 avenue Pierre Mendes France, 69500 Bron

n.guidou@gmail.com 